\title{
ORIGINAL
}

\section{Proximal nail fold flap for digital mucous cyst excision}

\author{
Yutaro Yamashita ${ }^{1}$, Hiroaki Nagae ${ }^{2}$, Ryosuke Yamato $^{2}$, Hiromichi Sedo ${ }^{3}$, Yoshiro Abe $^{1}$, and Ichiro Hashimoto ${ }^{1}$ \\ ${ }^{1}$ Department of Plastic and Reconstructive Surgery, Institute of Biomedical Sciences, Tokushima University Graduate School, Tokushima, Japan, \\ ${ }^{2}$ Department of Plastic Surgery, Tokushima Red Cross Hospital, Komatsushima, Japan, ${ }^{3}$ Department of Plastic Surgery, Tokushima Prefectural \\ Central Hospital, Tokushima, Japan
}

\begin{abstract}
The skin covering a digital mucous cyst is often very thin and is often excised with the cyst. Thus, transfer of a skin flap is needed for the defect. We have developed a proximal nail fold flap technique by which the thin skin covering the cyst can be preserved. We conducted a retrospective study to assess the effectiveness and reliability of this technique for digital mucous cyst excision. The study group comprised 26 patients treated for 28 digital mucous cysts. The flap was elevated on the nail matrix to expose the distal interphalangeal joint capsule. To preserve the skin in cases in which the skin covering the cyst was exceptionally thin, we did not excise the upper part of the cyst wall. Excision of the cyst and stalk was successful in all cases. Additional excision of the joint capsule or osteophyte(s) was achieved in 20 cases and 5 cases, respectively. No flap necrosis, skin defect or nail deformity resulted. Three of the cysts recurred and were treated successfully by reoperation involving the same flap elevation technique. We conclude that the proximal nail fold flap is useful for excision and reliable for wound coverage after digital mucous cyst excision. J. Med. Invest. 63 : 278-280, August, 2016
\end{abstract}

Keywords : ganglion cysts, osteophyte, finger joint, joint capsule

\section{INTRODUCTION}

Digital mucous cyst is a lesion that is commonly found in association with osteoarthritis of a distal interphalangeal (DIP) joint or thumb interphalangeal (IP) joint. Such cysts are sometimes painful and interfere with a person's daily activities. When the skin covering the cyst at the proximal nail fold is very thin and easily torn, the skin is often excised along with the cyst, and a skin flap or skin graft is transferred to the skin defect $(1,2)$. Patients must rest the finger for several days after this surgery. We developed and have been using a proximal nail fold flap technique for surgical excision of a digital mucous cyst associated with a DIP joint or thumb IP joint since 2006 (3). In the present study, comparing with the Japanese paper (3), we excluded patients who underwent simple cyst excision without stalk and joint capsule excision before 2011 and added patients who underwent stalk, joint capsule or osteophyte excision after 2012 in order to study effectiveness of this surgical technique for access to the joint capsule. Despite its thinness, the skin covering the cyst is not excised and is used to as a flap cover the wound. To determine the effectiveness and reliability of this skin flap technique, we retrospectively evaluated postoperative outcomes in patients who underwent the procedure. We report these outcomes along with a description of the technique itself.

\section{METHODS}

\section{Patient group}

The study group comprised 26 patients treated for 28 digital mucous cysts between July 2012 and April 2015. The study was approved by the Ethics Committee of Tokushima University Hospital (No. 2602). Twenty-two of the cysts were located at a DIP, and six

Received for publication March 14, 2016 ; accepted July 10, 2016.

Address correspondence and reprint requests to Ichiro Hashimoto, MD, $\mathrm{PhD}$, Department of Plastic and Reconstructive Surgery, Institute of Biomedical Sciences, Tokushima University Graduate School, 3-18-15 Kuramoto, Tokushima, 770-8503, Japan and Fax : +81-88-633-7297. were located at a thumb IP. Thirteen of the patients were men, and 13 were women ; age ranged from 49 to 83 years (mean, 63.6 years).

\section{Evaluation}

We were granted, by our respective surgical departments, access to the surgical records of the 26 patients, and we reviewed these records for the immediate surgical outcomes and any postoperative complications such as skin flap necrosis, a skin defect or nail deformity, joint stiffness or loss of motion, and recurrence. The patients had been followed up for a mean of 7.8 months (range, 3-19 months).

\section{Surgical Technique}

In all patients, the surgery was performed under digital nerve block achieved with $1 \%$ lidocaine, and a digital tourniquet was placed. Two skin incisions were made, 1 on each side of the proximal nail fold toward the midlateral line of the digit and approaching the DIP or IP joint (Figure 1). The skin flap was elevated on the nail matrix and under the cyst wall to expose the DIP joint capsule (Figure 2). In most cases, the cyst and stalk attached under the flap were carefully excised from the flap with a scalpel or scissors (Figure 3). If the skin on the cyst was exceptionally thin, we preserved it by not excising the upper part of the cyst wall. The cyst and stalk were easily identified and excised in the wide field that resulted from the flap elevation. When the stalk of the cyst was connected to the joint capsule, a part of the capsule was excised. Any offending osteophyte seen on the X-ray film was excised. The flap was then returned to its proper position and sutured into place. We permitted patients to use the finger whenever the wound pain had subsided.

\section{RESULTS}

All 28 cysts were excised with their stalks. Additional joint capsule excision and osteophyte excision were performed for 13 of the 28 cysts and 5 of the 28 cysts, respectively. 


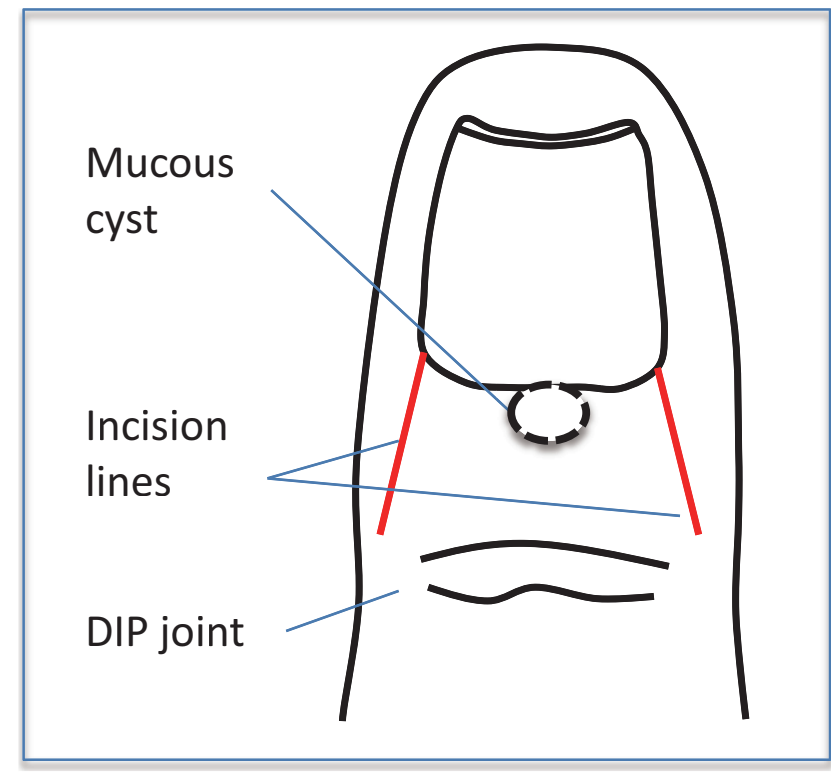

Figure 1. Design of the proximal nail fold flap.

Two skin incision lines are drawn from the proximal nail fold toward the midlateral line of the digit and approaching the DIP or IP joint. Note that the flap base is wider than the distal edge of the flap.

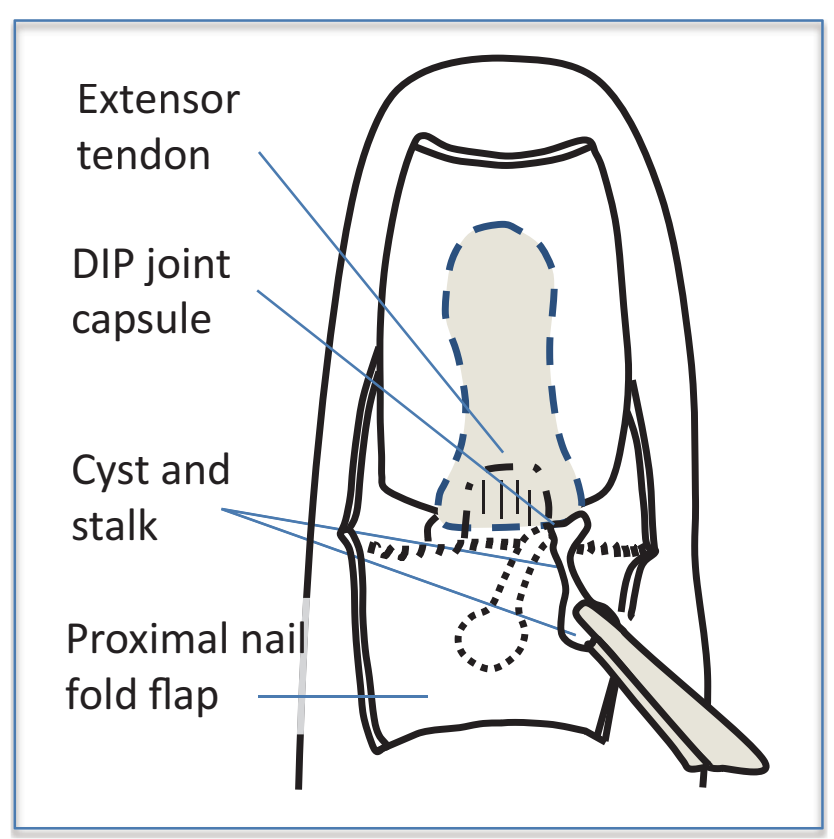

Figure 3. Diagram of the step taken after flap elevation. The cyst and stalk connected to the DIP joint are held and excised.

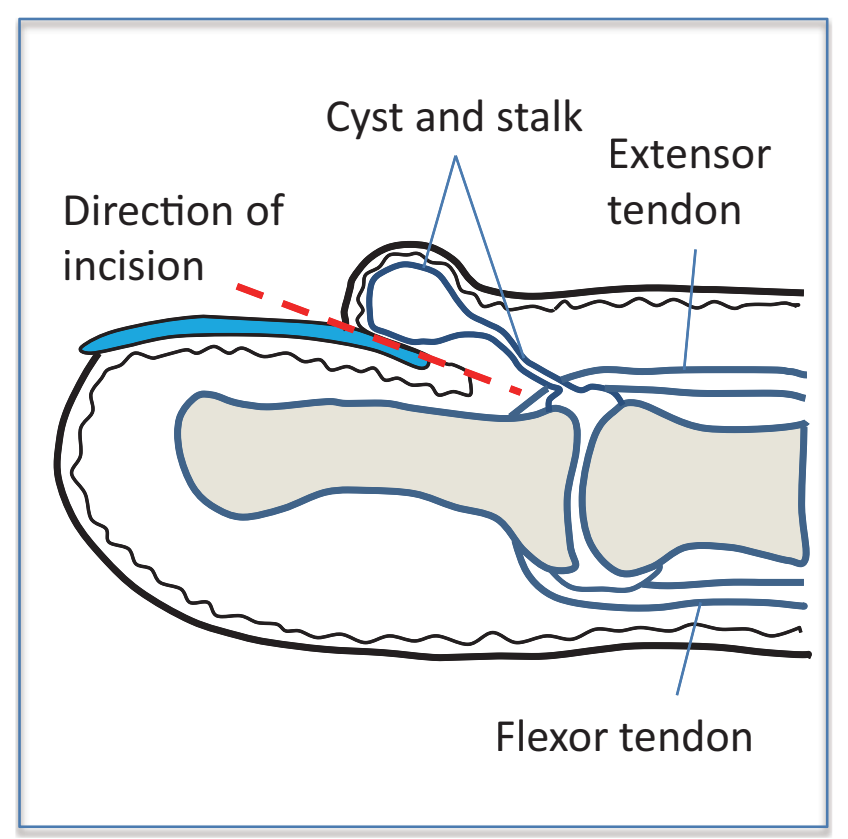

Figure 2. Cross-sectional diagram of the surgical technique. The flap under the mucous cyst is elevated along with the cyst. (Reproduced from Yamashita et al. (3), with permission.)

No flap necrosis, skin defect or nail deformity resulting from elevation of the proximal nail fold flap occurred in our patient group (Figure 4). Nail deformity was present before surgery in 9 of the 28 digits (32\%), and it disappeared postoperatively in 7 digits (77.8\%). Postoperative complications included the following : deformity of the proximal nail fold in 1 patient, tenderness in 1 patient who underwent joint capsule excision, and slight stiffness of the DIP joint in 2 patients who underwent osteophyte excision. There were 4 recurrences. The recurrence rates were $40 \%(4 / 10)$ in cyst and stalk excision, $0 \%(0 / 13)$ in capsule excision, and $0 \%(0 / 5)$ in osteophyte. The part of the cyst under very thin skin was not excised in 5 digits, and the cyst recurred in 1 of these cases. Reoperation involving the same flap elevation technique was performed for 3 of the 4 recurrent cysts. Joint capsule excision was performed during all 3 reoperations, and there was no postoperative complication or further recurrence.

\section{DISCUSSION}

Although treatment strategies for digital mucous cyst remain controversial $(1,2,4,5)$, excision of such cysts is performed commonly, and new methods that can simplify the procedure and reduce complications and recurrence are desired. In applying our proximal nail fold flap technique, we found that excision of the stalk, joint capsule and any osteophyte was easily achieved under the wide operative field obtained after elevation of the flap. Additionally, in no case did this flap result in a skin defect, and no additional skin flap or skin graft was required. This is because the blood circulation of the flap is not impaired, and even very thin skin covering the cyst is preserved with a part of the cyst wall.

Various surgical incisions, such as the $\mathrm{T}$ incision and $\mathrm{H}$ incision, are reported for excision of a digital mucous cyst (6). An advantage of the proximal nail fold flap is that the operative field under the proximal nail fold flap is wider than it is under the $\mathrm{T}$ or $\mathrm{H}$ incision. Elevation of a narrow skin flap on the mucous cyst was reported in 2005 (5). However, this flap exposes only the ipsilateral side of the DIP joint. Under the proximal nail fold flap, both sides of the DIP joint can be approached. This makes identifying the attachment of the extensor tendon, DIP joint capsule and osteophyte easy. This identification is essential for proper excision of the stalk, joint capsule and osteophyte(s). The recurrence rates of cyst and stalk excision, capsule excision and osteophyte were almost same as the recurrence rates in past reports $(4,5)$. 

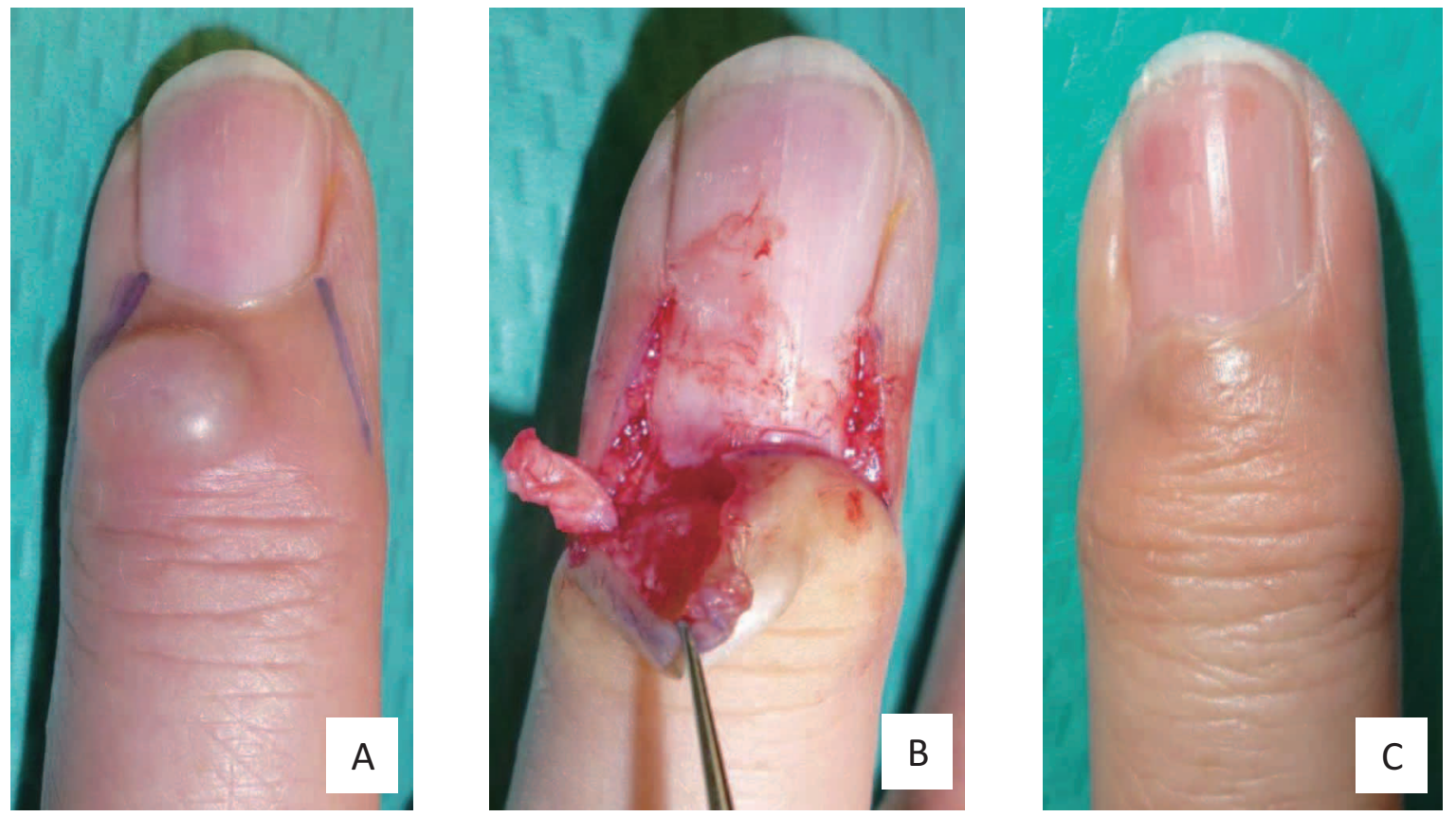

Figure 4. Preoperative and postoperative photograph.

Photographs of a representative case showing the cyst and skin incision lines just before the surgery (A), elevated flap and exposed cyst (B) and the healed digit 6 months after surgery (C).

Few complications occurred in our patient group. Traumatic elevation of the flap may have caused the proximal nail fold deformity experienced by 1 patient and the tenderness experienced by another patient. Non-traumatic procedures are essential if we wish to avoid complications around the nail and joint. Stiffness of the DIP joint occurred only in patients who underwent osteophyte excision. We think that the joint stiffness was related to invasion of the joint rather than the flap elevation.

The resulting proximal nail fold flap scar in each of our patients was deemed aesthetically acceptable. We were also quite pleased that this flap could be elevated twice along the same lines of incision in cases of relapse. We conclude that this proximal nail fold flap technique is quite useful for digital mucous cyst excision.

\section{CONFLICT OF INTEREST}

None.

\section{FUNDING}

No special funding.

\section{REFERENCES}

1. Jamnadas-Khoda B, Agarwal R, Harper R, Page RE : Use of Wolfe graft for the treatment of mucous cysts. J Hand Surg Eur 34 : 519-521, 2009

2. Imran D, Koukkou C, Bainbridge LC : The rhomboid flap : a simple technique to cover the skin defect produced by excision of a mucous cyst of a digit. J Bone Joint Surg 85B : 860862, 2003

3. Yamashita Y, Nagae H, Yamato R: Mucous cyst excision by the proximal nail fold-elevating technique. J Jpn Plast Reconstr Surg 34 : 869-874, 2014

4. Eaton RG, Dobranski AI, Littler JW : Marginal osteophyte excision in treatment of mucous cysts. J Bone Joint Surg 55A : 570-574, 1973

5. Lawrence $\mathrm{C}$ : Skin excision and osteophyte removal is not required in the surgical treatment of digital myxoid cysts. Arch Dermatol 141: 1560-1564, 2005

6. Brown RE, Zook EG, Russell RC, Kucan JO, Smoot EC : Fingernail deformities secondary to ganglions of the distal interphalangeal joint (mucous cysts). Plast Reconstr Surg 87 : 718 725,1991 